\title{
Human Fibroblasts Maintain the Viability and Augment the Functional Response of Human Neutrophils in Culture
}

\author{
Carolyn J. Ling, William F. Owen, Jr., and K. Frank Austen \\ Department of Medicine, Harvard Medical School, and Department of Rheumatology and Immunology, \\ Brigham and Women's Hospital, Boston, Massachusetts 02115
}

\begin{abstract}
When human neutrophils were co-cultured for $72 \mathrm{~h}$ with nontransformed human fibroblasts, $69 \pm 3 \%(n=13)$ survived, as compared with survival levels of $2 \pm 1 \%(n=15)$ and $26 \pm 6 \%$ $(n=7)$, respectively, for neutrophils cultured for the same time period in enriched medium alone or supplemented with 10 pM recombinant human granulocyte/macrophage colony-stimulating factor (rh GM-CSF). Conditioned medium from the human fibroblast cultures enhanced neutrophil survival in a dose-dependent fashion to the same level achieved with neutrophil/fibroblast co-cultures, and its soluble viability-sustaining activity was not inhibited by preincubation with neutralizing antiserum against rh GM-CSF. As compared with freshly isolated replicate samples, neutrophils co-cultured with human fibroblasts for $72 \mathrm{~h}$ exhibited augmented FMLP-stimulated superoxide production without spontaneous superoxide generation. This striking extension of survival and associated priming for a ligand response by neutrophils co-cultured with human fibroblasts suggests that fibroblasts may contribute to the proinflammatory properties of neutrophils in tissues. (J. Clin. Invest. 1990. 85:601-604.) polymorphonuclear leukocyte $\bullet$ cytokine - granulocyte - connective tissue - superoxide • colony-stimulating factor
\end{abstract}

\section{Introduction}

Neutrophils released from the bone marrow exist in freely exchangeable intravascular circulating and marginated pools and in an extravascular pool within the tissue microenvironment (1). The survival of diisopropylfluorophosphate-labeled human neutrophils within the circulation and the marginated pool is brief with a half-life of 6-7 h (2). There are no data on the lifespan of human neutrophils in tissues. Recent studies that have demonstrated the ability of granulocyte/macrophage colony-stimulating factor (GM-CSF) ${ }^{1}$ and granulocyte col-

Address reprint requests to Dr. Ling, The Seeley G. Mudd Building, Room 610, 250 Longwood Avenue, Boston, MA 02115.

Received for publication 30 August 1989 and in revised form 14 November 1989.

1. Abbreviations used in this paper: G-CSF, granulocyte colony-stimulating factor; GM-CSF, granulocyte/macrophage colony-stimulating factor, HBSS $^{*}$, Hanks' balanced salt solution without calcium or magnesium; rh, recombinant human.

J. Clin. Invest.

(C) The American Society for Clinical Investigation, Inc.

$0021-9738 / 90 / 02 / 0601 / 04 \$ 2.00$

Volume 85, February 1990, 601-604 ony-stimulating factor (G-CSF) to prime or to directly enhance various neutrophil functions have not specifically examined the ability of these cytokines simultaneously to extend neutrophil viability ex vivo (3-6). GM-CSF, the only cytokine that has been reported to enhance neutrophil survival ex vivo, extended the half-life for neutrophil survival from $22 \mathrm{~h}$ in its absence to $28 \mathrm{~h}$ in the presence of $\sim 0.2 \mathrm{pM} \mathrm{GM-CSF} \mathrm{(7).}$

We have previously reported that human eosinophils cultured with endothelial cells, with their conditioned medium, or with certain cytokines remain viable and exhibit augmented functional responses to a separate stimulus (8-11). We now report that the survival of neutrophils cultured for $72 \mathrm{~h}$ in enriched medium supplemented with $10 \mathrm{pM}$ GM-CSF increased from $2 \%$ when cultured in enriched medium alone to $26 \%$, whereas the survival of neutrophils co-cultured with a human fibroblast line (HF-15) for the same time period increased to $69 \%$. Furthermore, the fibroblast-derived, viabilitysustaining activity was soluble and was not neutralized by antiserum to GM-CSF. As compared with freshly isolated replicate samples, neutrophils that had been co-cultured for $72 \mathrm{~h}$ with fibroblasts had no constitutive superoxide production but generated twofold greater amounts of superoxide when stimulated with FMLP. Thus, a fibroblast component has the dual effects of increasing neutrophil viability and of priming the neutrophils for a ligand-specific response. This culture system, which does not involve adherence or "activation" as defined by spontaneous superoxide generation, may provide a cell source suitable for in vitro studies of human peripheral blood neutrophils for 72 or more hours after harvest.

\section{Methods}

Establishment of neutrophil/human fibroblast co-cultures. Passages number 10-12 of human HF-15 fibroblasts (kindly provided by Dr. J. Moss, National Institutes of Health, Bethesda, MD) were established in enriched medium (RPMI 1640 culture medium [Gibco Laboratories, Grand Island, NY], 10\% fetal bovine serum, 2 mM L-glutamine, 0.1 $\mathrm{mM}$ nonessential amino acids [Sigma Chemical Co., St. Louis, MO], $100 \mathrm{U} / \mathrm{ml}$ penicillin, $100 \mu \mathrm{g} / \mathrm{ml}$ streptomycin, and $10 \mu \mathrm{g} / \mathrm{ml}$ gentamycin) by plating in $75 \mathrm{~cm}^{2}$ plastic tissue culture flasks. After reaching confluence, the fibroblasts were passaged by treatment with trypsin and were reseeded in $2 \mathrm{ml}$ of enriched medium onto $35-\mathrm{mm}$ culture dishes at a density of $5 \times 10^{4}$ cells $/ \mathrm{ml}$. Confluence was achieved again after 7-10 d and corresponded to $3-4 \times 10^{5}$ fibroblasts.

Neutrophils were obtained from the peripheral blood of healthy volunteers by sedimentation with dextran (Dextran T500; Pharmacia Fine Chemicals, Piscataway, NJ), gradient centrifugation with FicollHypaque (Pharmacia Inc.), and lysis of erythrocytes with hypotonic sodium chloride (12). The final cell preparations routinely consisted of $>97 \%$ neutrophils. Eosinophils were the sole contaminating granulocyte. Freshly isolated neutrophils $\left(0.5-2 \times 10^{6}\right.$ cells $)$ in $2 \mathrm{ml}$ of 
enriched medium were added to the confluent fibroblast culture, and the medium of each dish was supplemented every $48 \mathrm{~h}$ by the addition of $1 \mathrm{ml}$ of fresh enriched medium.

In other experiments, replicate confluent fibroblast cultures were washed with Hanks' balanced salt solution without calcium or magnesium $\left(\mathrm{HBSS}^{-}\right)$and incubated with buffered $2 \%$ (vol/vol) paraformaldehyde for $15 \mathrm{~min}$ at room temperature. The fixed fibroblast monolayers were washed three times with $2 \mathrm{ml}$ of $\mathrm{HBSS}^{*}$, and neutrophil cultures were performed as described above.

Fibroblast-conditioned medium was aspirated from confluent cultures of HF- 15 fibroblasts. The conditioned medium was pooled, centrifuged for $30 \mathrm{~min}$ at $1,000 \mathrm{~g}$, filtered through $0.45-\mu \mathrm{m}$ filters, and stored for up to $7 \mathrm{~d}$ at $-20^{\circ} \mathrm{C}$. The conditioned medium was concentrated to a $10 \times$ stock by filtration under positive pressure through a YM-10 membrane in an Amicon concentrator (size exclusion of 10,000 D) (Amicon Filter Corp., New Bedford, MA), and stored at $-20^{\circ} \mathrm{C}$. In some experiments, neutrophils were cultured in $2 \mathrm{ml}$ of enriched medium alone or in enriched medium supplemented with incremental doses of recombinant human (rh) GM-CSF (provided by Dr. S. Clark, Genetics Institute, Boston, MA), rh interleukin 3 (IL-3) (provided by Dr. Y.-C. Yang, Genetics Institute), rh G-CSF (Genzyme Inc., Boston, MA), or combinations of either GM-CSF with IL-3 or G-CSF. At 48-h intervals, $1 \mathrm{ml}$ of fibroblast-conditioned medium or cytokine-supplemented enriched medium was added as described above for the fibroblast co-cultures. In separate experiments, neutrophils were cultured in conditioned medium that had been prepared as usual or heated to temperatures of $65^{\circ}$ or $80^{\circ} \mathrm{C}$, respectively, for $1 \mathrm{~h}$.

Analysis of neutrophil survival. At the designated time, neutrophils were recovered by rinsing each culture dish with $10 \mathrm{ml}$ of enriched medium. The neutrophil suspensions were pooled and centrifuged at $250 \mathrm{~g}$ for $5 \mathrm{~min}$ at $25^{\circ} \mathrm{C}$, and the pellets were resuspended in $1 \mathrm{ml}$ of enriched medium. Cell numbers were determined in a Neubauer hemacytometer, and cell viability was assessed by trypan blue exclusion. Differential cell counts were determined after staining with Wright's and Giemsa. The survival of cultured neutrophils was calculated as follows: $100 \times$ [(total number of cells remaining) $\times$ (percent of cells excluding trypan blue) $\times$ (percent neutrophils as per Wright-Giemsa stain)]/[original number of cells seeded $\times$ percent neutrophils].

Antibody neutralization assay. In individual wells of a 96-well flatbottomed microtiter plate, 50- $\mu \mathrm{l}$ aliquots of a 1:10 dilution of 10X stock fibroblast-conditioned medium were preincubated for $2.5 \mathrm{~h}$ at $37^{\circ} \mathrm{C}$ with $50 \mu \mathrm{l}$ of enriched medium alone or with an equal volume of neutralizing rabbit polyclonal antiserum to th GM-CSF (50 $\mu \mathrm{g})(\mathrm{Gen}$ zyme Inc.). $10^{5}$ neutrophils in $100 \mu$ l of enriched medium were added to each well, and the incubations were continued for $72 \mathrm{~h}$ more without the further addition of medium. The neutrophils were then counted and assessed for viability by trypan blue exclusion. Replicate neutrophils were incubated in enriched medium alone, medium supplemented with $50 \mathrm{pM}$ rh GM-CSF, or medium supplemented with 50 pM rh GM-CSF and antiserum to rh GM-CSF.

Superoxide production. Freshly isolated or cultured neutrophils were suspended at a density of $6.25 \times 10^{5} \mathrm{cells} / \mathrm{ml}$ in Hanks' balanced salt solution with calcium and magnesium, $5 \%$ fetal bovine serum, $0.1 \%$ bovine serum albumin, and $1 \mathrm{mM}$ Hepes. Samples $(800 \mu \mathrm{l})$ of this cell suspension were combined with $100 \mu \mathrm{l}$ of freshly prepared cytochrome $c$ (Sigma Chemical Co.; type V, $10 \mathrm{mg} / \mathrm{ml}$ ) in HBSS" with or without the addition of $20 \mu$ l of superoxide dismutase (Sigma Chemical Co., $1 \mathrm{mg} / \mathrm{ml}$ ) in HBSS $^{=}$, and the tubes were placed in a shaking water bath at $37^{\circ} \mathrm{C}$ for $5 \mathrm{~min}$. Various concentrations of FMLP were added to produce a final volume of $1 \mathrm{ml}$, and incubations were continued for $10 \mathrm{~min}$. The reactions were terminated by rapidly cooling the samples and centrifuging them at $1,000 \mathrm{~g}$ for $30 \mathrm{~min}$ at $4^{\circ} \mathrm{C}$. The supernatants were immediately transferred to cuvettes for measurement of reduced cytochrome $c$ at an absorbance of $550 \mathrm{~nm}$ using an extinction coefficient of $18.5 \mathrm{mM}^{-1}$.

Statistical analysis. Data are expressed as the mean \pm SEM for experiments performed three times or more, and as the mean $\pm 1 / 2$ range for experiments performed twice. Means were compared by Student's $t$ test in a two-tailed analysis.

\section{Results}

Effect of co-culture with HF-15 human fibroblasts on neutrophil survival. Freshly isolated human neutrophils were maintained in enriched medium alone or in medium supplemented with incremental concentrations of rh GM-CSF. In enriched medium alone, $2 \pm 1 \%(n=15)$ of the neutrophils survived for $72 \mathrm{~h}$. The addition of $\mathrm{rh}$ GM-CSF at doses of $1-100 \mathrm{pM}$ to the culture medium augmented neutrophil survival in a dose-dependent fashion to a maximal survival of $26 \pm 6 \%(n=7, P$ $<0.001$ ) at $72 \mathrm{~h}$ in the presence of $10 \mathrm{pM}$ rh GM-CSF.

Because murine 3T3 fibroblasts have been shown to extend the survival of purified rat serosal mast cells (13), dispersed human lung mast cells (14), and human peripheral blood eosinophils exposed to cytokines (9), we examined their ability and that of HF-15 human fibroblasts to augment neutrophil survival ex vivo. Only $11 \pm 3 \%(n=3)$ of the neutrophils survived for $72 \mathrm{~h}$ when co-cultured with mouse $3 \mathrm{~T} 3$ fibroblasts alone, as compared with $9 \pm 4 \%$ for replicate neutrophils maintained in enriched medium alone $(P>0.1)$. However, survival for replicate neutrophils co-cultured for $72 \mathrm{~h}$ with HF-15 human fibroblasts increased to $80 \pm 4 \%(P<0.01)$. Furthermore, by light microscopy, neutrophils co-cultured for $72 \mathrm{~h}$ with HF-15 fibroblasts were unchanged morphologically from freshly isolated replicate neutrophils. Mitotic figures were not observed.

The time course for the enhancement of neutrophil survival by co-culture with HF-15 fibroblasts was statistically significant at each of the 24-h intervals examined (Fig. 1). When neutrophils were cultured for $72 \mathrm{~h}$ in enriched medium alone or in the presence of $10 \mathrm{pM}$ rh GM-CSF, 1 and $15 \%$ of the original cells survived, respectively, as compared with $78 \%$ survival for neutrophils co-cultured with HF-15 fibroblasts. When neutrophils were co-cultured with HF-15 fibroblasts fixed in paraformaldehyde, cell survival was reduced to $3 \pm 2 \%$ $(n=3)$ at $72 \mathrm{~h}$ as compared with $7 \pm 0 \%$ and $63 \pm 4 \%$ for replicate neutrophils maintained in enriched medium alone or co-cultured with viable HF-15 fibroblasts, respectively.

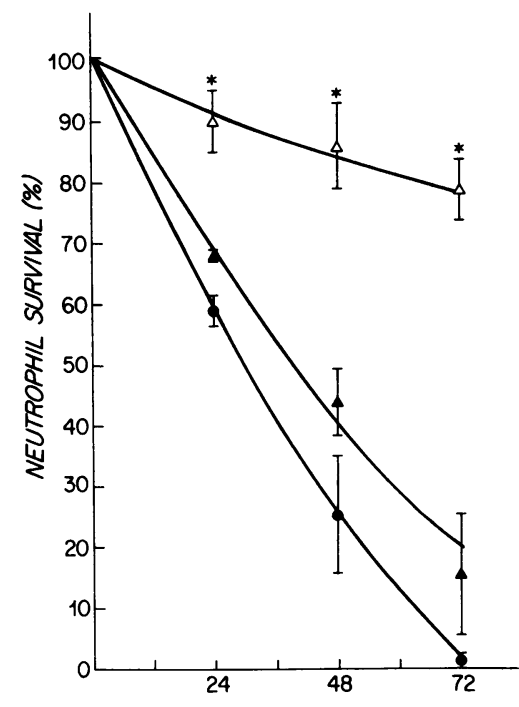

Figure 1. Time course for the survival of freshly isolated neutrophils cultured in enriched medium alone $(\bullet)$ or supplemented with $10 \mathrm{pM}$ rh GMCSF ( $\Delta)$, or co-cultured with HF-15 human fibroblasts $(\Delta)$. Values shown represent the mean \pm SEM for each culture condition ( $n$ $=3$ ). * Refers to a statistically significant increase in neutrophil survival as compared with cells maintained in medium alone $(P<0.01)$. 
Effect of defined cytokines or HF-15 human fibroblast-conditioned medium on neutrophil survival. The conditioned medium from the HF-15 fibroblasts sustained neutrophil survival ex vivo in a dose-dependent manner (Fig. 2). The neutrophil survival of $66 \pm 9 \%(n=3)$ observed after $72 \mathrm{~h}$ using a 1:10 dilution of $10 \times$ stock fibroblast-conditioned medium was comparable to that of replicate neutrophils in HF-15 fibroblast co-cultures, which demonstrated $73 \pm 8 \%$ survival $(P>1.0)$. When replicate samples of neutrophils were cultured for $72 \mathrm{~h}$ in $10 \times$ stock enriched medium alone at dilutions even as low as $1: 2$, less than $2 \%$ of the cells survived. To determine the heat stability of the viability enhancing activity in fibroblast-conditioned medium, 1:10 dilutions of $10 \times$ stock conditioned medium were heated for $1 \mathrm{~h}$ at either $65^{\circ} \mathrm{C}$ or $80^{\circ} \mathrm{C}$. Conditioned medium that had been heated at either $65^{\circ} \mathrm{C}$ or $80^{\circ} \mathrm{C}$ did not augment neutrophil survival whereas 72 -h survival of neutrophils cultured in conditioned medium kept at $4^{\circ} \mathrm{C}$ before assay was $63 \%$ as compared to $2 \%$ in enriched medium alone.

Because of the limited viability enhancing activity of GMCSF alone, this cytokine was studied in combination with IL-3 or G-CSF. One cytokine was held at a fixed dose and the other was included at incremental concentrations; survival of neutrophils was assessed at $72 \mathrm{~h}$. A combination of $10 \mathrm{pM}$ rh IL-3 with increasing concentrations of rh GM-CSF (0.1-10 pM) was unable to maintain the 72-h survival of neutrophils above that achieved with replicate samples that were cultured in rh GM-CSF alone ( $8 \%$ at $10 \mathrm{pM})$. Culture of neutrophils with a fixed concentration of $10 \mathrm{pM}$ rh GM-CSF and increasing concentrations of rh IL-3 (0.1 to $10 \mathrm{pM})$ did not enhance $72-\mathrm{h}$ survival above that achieved with $10 \mathrm{pM}$ rh GM-CSF alone (28\%). The positive control of a 1:10 dilution of $10 \times$ stock HF-15 fibroblast-conditioned medium had a survival of $50 \%$ after $72 \mathrm{~h}$ of culture as compared to $2 \%$ for enriched medium with or without $10 \mathrm{pM}$ rh IL-3.

In contrast, neutrophils cultured with a fixed concentration of $50 \mathrm{U}$ of rh G-CSF and increasing concentrations of rh GM-CSF demonstrated a dose-dependent enhancement of survival at $72 \mathrm{~h}$ to $22 \%$, as compared with $8 \%$ with $10 \mathrm{pM}$ rh GM-CSF alone, $2 \%$ with enriched medium alone, and $2 \%$ with $50 \mathrm{U}$ of rh G-CSF alone. Furthermore, neutrophils cultured with $10 \mathrm{pM}$ rh GM-CSF and increasing concentrations of $\mathrm{rh}$ G-CSF (50-200 U) demonstrated a dose-dependent enhancement of survival at $72 \mathrm{~h}$ to $55 \%$ for $200 \mathrm{U}$ of rh G-CSF as compared to $25 \%$ for this concentration of G-CSF alone, $28 \%$ with 10 pM GM-CSF alone, and $2 \%$ in enriched medium alone. The positive control of a 1:10 dilution of 10× stock conditioned medium had a 72 -h survival of $76 \%$.

Because stimulated human fibroblasts elaborate GM-CSF

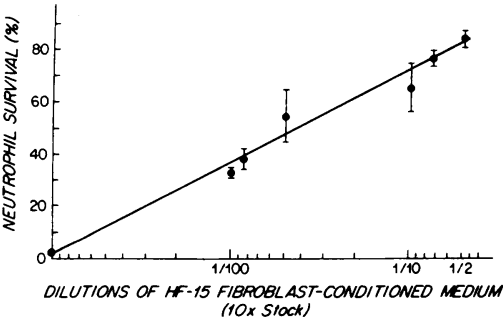

Figure 2. Influence of incremental dilutions of 10X stock HF- 15 fibroblast-conditioned medium on the survival of human neutrophils. Survival was determined after $72 \mathrm{~h}$ in culture. Values shown represent the mean \pm SEM for triplicate experiments.
(15) and this cytokine is supplemented in activity by G-CSF, we examined the ability of polyclonal rabbit anti-human GMCSF to inhibit the soluble fibroblast-derived, viability-sustaining activity of the HF-15 fibroblast-conditioned medium. The inclusion of $50 \mu \mathrm{g}$ of anti-GM-CSF with $100 \mathrm{pM}$ rh GM-CSF reduced neutrophil viability after $3 \mathrm{~d}$ from $33 \pm 18 \%$ to $8 \pm 3 \%$ ( $n=2$; mean $\pm 1 / 2$ range), which was similar to the $14 \pm 4 \%$ survival of the cells in enriched medium alone. In contrast, when replicate neutrophils were incubated for $72 \mathrm{~h}$ with a 1:10 dilution of $10 \times$ stock fibroblast-conditioned medium alone or in the presence of $50 \mu \mathrm{g}$ anti-GM-CSF, $39 \pm 6 \%$ and $45 \pm 6 \%$ survived, respectively.

Superoxide generation by co-cultured neutrophils. In three experiments in which freshly isolated neutrophils were stimulated with incremental concentrations of FMLP, optimal reduction of cytochrome $c$ was observed at an FMLP dose of $10^{-6} \mathrm{M}\left(14 \pm 1 \mathrm{nmol} / 5 \times 10^{5}\right.$ neutrophils), and an $\mathrm{ED}_{50}$ of 1.8 $\times 10^{-7}$ M FMLP was extrapolated (Fig. 3). Replicate neutrophils co-cultured for $72 \mathrm{~h}$ with HF-15 fibroblasts reduced a maximum of $29 \pm 2 \mathrm{nmol}$ of cytochrome $c / 5 \times 10^{5}$ cells when stimulated with $10^{-6} \mathrm{M}$ FMLP, and the extrapolated $\mathrm{ED}_{50}$ was $0.5 \times 10^{-7} \mathrm{M}$ FMLP. Neither freshly isolated nor co-cultured neutrophils generated detectable amounts of superoxide in the absence of FMLP stimulation, and superoxide dismutase completely abolished the FMLP-stimulated reduction of cytochrome $c$ (data not shown).

\section{Discussion}

Studies of neutrophil function ex vivo have relied upon neutrophils isolated from the circulating blood pool, whose survival is generally quite poor (half-life $<6-7 \mathrm{~h}$ ) (2). Using $\sim 0.2$ pM GM-CSF, Lopez et al. (7) were able to extend the median survival of $50 \%$ of the originally plated cells by only $6 \mathrm{~h}$ (from 22 to $28 \mathrm{~h}$ ). We have found that an optimal concentration of 10 pM GM-CSF extended survival for $50 \%$ of the original neutrophils from $28 \mathrm{~h}$ to $\sim 38 \mathrm{~h}$. rh IL-3 alone had no effect on neutrophil survival and did not augment survival above that observed with rh GM-CSF. In contrast, rh G-CSF, which had some minimal viability enhancing activity at $50 \mathrm{U}$ alone

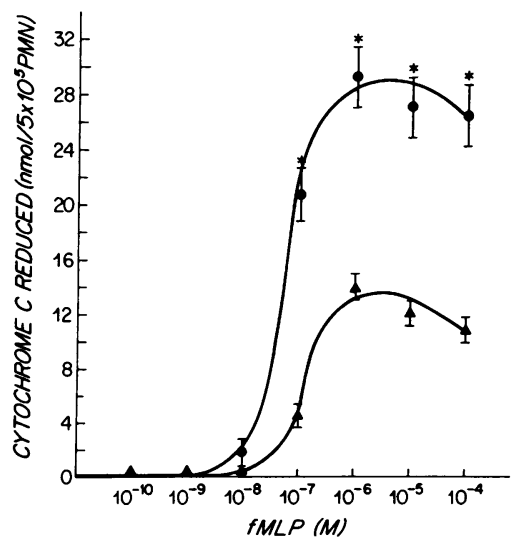

Figure 3. Effect of incremental doses of FMLP on superoxide generation by freshly isolated neutrophils $(\Delta)$ and replicate neutrophils co-cultured for 72 $h$ with HF-15 fibroblasts (๑). Cytochrome $c$ reduction was monitored at $550 \mathrm{~nm}$ for 5 min at $37^{\circ} \mathrm{C}$. Values shown represent the mean \pm SEM for triplicate experiments. $*$ Statistically significant increase in FMLP-stimu-

lated superoxide generation as compared to that of freshly isolated replicate neutrophils $(P<0.05)$. 
(mean $7 \pm 4 \%, n=4$ ), did augment the viability of cells cultured in combination with rh GM-CSF in a dose-dependent manner. However, the combination of rh GM-CSF and rh G-CSF at a plateau response sustained fewer neutrophils viable at $72 \mathrm{~h}$ than the 1:10 dilution of $10 \times$ stock-conditioned medium from HF-15 fibroblasts.

For all experiments, co-culture of the noncontact inhibited, nontransformed human HF-15 fibroblast line with neutrophils resulted in a dramatic increase in neutrophil survival at $72 \mathrm{~h}$ to $69 \pm 3 \%(n=13)$, which was 3-fold the percentage surviving in $10 \mathrm{pM}$ rh GM-CSF $(n=4)$, and $\sim 10$-fold the percentage surviving with $50 \mathrm{U}$ rh G-CSF $(n=4)$. As evidenced by the decreased slope for the loss of viability over the 72-h period of culture, the time-dependent spontaneous loss of viability for the entire population maintained in enriched medium with rh GM-CSF was attenuated (Fig. 1). HF-15 fibroblasts fixed in paraformaldehyde did not maintain neutrophil viability, indicating that an interaction between the neutrophils and the fibroblast-derived extracellular matrix was not adequate. In contrast, conditioned medium from the HF-15 fibroblasts was equally capable of maintaining neutrophil survival in a dose-dependent fashion (Fig. 2). Neutralizing antiserum to rh GM-CSF was unable to abolish the viability-sustaining activity of the fibroblast-conditioned medium, but fully neutralized the viability-sustaining activity of rh GMCSF-supplemented enriched medium. Thus, HF-15 human fibroblasts constitutively provide a soluble viability-enhancing factor for human neutrophils distinct from GM-CSF. These results also suggest that the enhancing activity of the conditioned medium does not reflect a combination of GM-CSF with G-CSF, since G-CSF alone was only modestly active as compared to the conditioned medium. Whether the activity of the conditioned medium represents a combination of G-CSF and some other factor or represents a distinctly different cytokine or set of cytokines is not resolved.

The co-cultured neutrophils did not exhibit spontaneous superoxide production but demonstrated a strikingly augmented capacity on a population basis to generate superoxide when stimulated with FMLP (Fig. 3). This response parallels previous reports of in vitro cytokine enhanced neutrophil oxidative metabolism, in particular of GM-CSF's ability to prime neutrophils to produce twofold greater amounts of superoxide on activation with FMLP (4). The capacity of human fibroblasts to elaborate soluble factors that can strikingly enhance neutrophil viability and ligand specific responses ex vivo suggests that fibroblasts may play a similar critical role in the tissue microenvironment for neutrophil-mediated host defense or tissue injury. Furthermore, this system of neutrophil/ fibroblast coculture without cellular adherence provides a simple means of optimizing neutrophil viability for in vitro biochemical studies.

\section{Acknowledgments}

We are grateful to Dr. Richard Jack and Marc Rothenberg for their helpful discussions.

This work was supported in part by grants AI-23401, AI-22531, AI-22563, AI-07306, HL-36110, AR-38638 from the National Institutes of Health; and in part by a grant from the Robert Wood Johnson Foundation (to Dr. Owen).

\section{References}

1. Craddock, C. G., Jr. 1960. The physiology of granulocytic cells in normal and leukemic states. Am. J. Med. 28:711-725.

2. Cartwright, G. E., J. W. Athens, and N. M. Wintrobe. 1964. The kinetics of granulopoiesis in normal man. Blood. 24:780-803.

3. Gasson, J. C., R. H. Weisbart, S. E. Kaufman, S. C. Clark, R. M. Harwick, G. G. Wong, and D. W. Golde. 1984. Purified human granulocyte-macrophage colony stimulating factor. Direct action on neutrophils. Science (Wash. DC). 226:1339-1342.

4. Weisbart, R. H., D. W. Golde, S. C. Clark, G. G. Wong, and J. C. Gasson. 1985. Human granulocyte-macrophage stimulating factor is a neutrophil activator. Nature (Lond.). 314:361-363.

5. Yuo, A., S. Kitagawa, T. Okabe, A. Urabe, Y. Komatsu, S. Itoh, and F. Takaku. 1987. Recombinant human granulocyte colony-stimulating factor repairs the abnormalities of neutrophils in patients with myelodysplastic syndromes and chronic myelogenous leukemia. Blood. 70:404-411.

6. Dahinden, C. A., J. Zingg, F. E. Maly, and A. L. deWeck. 1988. Leukotriene production in human neutrophils primed by recombinant human granulocyte/macrophage colony stimulating factor and stimulated with the complement component C5a and FMLP as second signals. J. Exp. Med. 167:1296-1312.

7. Lopez, A. F., J. Williamson, J. R. Gamble, C. G. Begley, J. M. Harlan, S. J. Klebanoff, A. Waltersdorph, G. Wong, S. C. Clark, and M. A. Vadas. 1986. Recombinant human granulocyte-macrophage colony-stimulating factor stimulates in vitro mature human neutrophil and eosinophil function, surface receptor expression, and survival. $J$. Clin. Invest. 78:1220-1228.

8. Rothenberg, M. E., W. F. Owen, Jr., D. S. Silberstein, R. J. Soberman, K. F. Austen, and R. L. Stevens. 1987. Eosinophils cocultured with endothelial cells have increased survival and functional properties. Science (Wash. DC). 237:645-647.

9. Owen, W. F., Jr., M. E. Rothenberg, D. S. Silberstein, J. C. Gasson, R. L. Stevens, K. F. Austen, and R. J. Soberman. 1987. Regulation of human eosinophil viability, density, and function by granulocyte/macrophage colony-stimulating factor in the presence of 3T3 fibroblasts. J. Exp. Med. 166:129-141.

10. Rothenberg, M. E., J. Petersen, R. L. Stevens, D. S. Silberstein, D. T. McKenzie, K. F. Austen, and W. F. Owen, Jr. 1989. Interleukin-5-dependent conversion of normodense human eosinophils to the hypodense phenotype uses 3T3 fibroblasts for enhanced viability, accelerated hypodensity, and sustained antibody-dependent cytotoxicity. J. Immunol. 143:2311-2316.

11. Rothenberg, M. E., W. F. Owen, D. S. Silberstein, J. Woods, R. J. Soberman, K. F. Austen, and R. L. Stevens. 1988. Human eosinophils have prolonged survival, enhanced functional properties, and become hypodense when exposed to human interleukin 3. J. Clin. Invest. 81:1986-1992.

12. Boyüm, A. 1968. Isolation of mononuclear cells and granulocytes from human blood. Scand. J. Clin. Lab. Invest. 21 (Suppl. 97):77-89.

13. Levi-Schaffer, F., K. F. Austen, J. P. Caulfield, A. Hein, W. Bloes, and R. L. Stevens. 1985. Fibroblasts maintain the phenotype and viability of rat heparin-containing mast cells in vitro. J. Immunol. 135:3454-3462.

14. Levi-Schaffer, F., K. F. Austen, J. P. Caulfield, A. Hein, P. M. Gravallese, and R. L. Stevens. 1987. Co-culture of human lung-derived mast cells with mouse 3T3 fibroblasts. Morphology and IgE mediated release of histamine, prostaglandin $D_{2}$, and leukotrienes. $J$. Immunol. 139:494-500.

15. Zucali, J. R., C. A. Dinarello, D. J. Oblon, M. A. Gross, L. Anderson, and R. S. Weiner. 1986. Interleukin 1 stimulates fibroblasts to produce granulocyte-macrophage colony stimulating activity and prostaglandin E2. J. Clin. Invest. 77:1857-1863. 